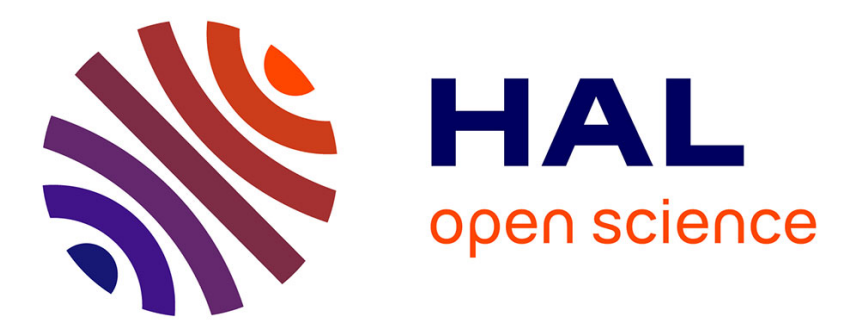

\title{
LA CULTURE DE MUTUALISATION DU PSL PEUT-ELLE FAVORISER L'ÉMERGENCE D'UNE LOGISTIQUE URBAINE DURABLE?
}

Odile Chanut, Paché Gilles

\section{- To cite this version:}

Odile Chanut, Paché Gilles. LA CULTURE DE MUTUALISATION DU PSL PEUT-ELLE FAVORISER L'ÉMERGENCE D'UNE LOGISTIQUE URBAINE DURABLE?. RIMHE: Revue Interdisciplinaire Management, Homme(s) \& Entreprise, 2013. hal-01767000

\section{HAL Id: hal-01767000 \\ https://hal.science/hal-01767000}

Submitted on 14 Apr 2018

HAL is a multi-disciplinary open access archive for the deposit and dissemination of scientific research documents, whether they are published or not. The documents may come from teaching and research institutions in France or abroad, or from public or private research centers.
L'archive ouverte pluridisciplinaire HAL, est destinée au dépôt et à la diffusion de documents scientifiques de niveau recherche, publiés ou non, émanant des établissements d'enseignement et de recherche français ou étrangers, des laboratoires publics ou privés. 


\title{
LA CULTURE DE MUTUALISATION DU PSL PEUT-ELLE FAVORISER L'ÉMERGENCE D'UNE LOGISTIQUE URBAINE DURABLE ?
}

\author{
Odile Chanut et Gilles Paché
}

\section{ARIMHE | RIMHE}

2013/3- $\mathrm{n}^{\circ} 7$

pages 94 à 110

ISSN 2259-2490

Article disponible en ligne à l'adresse:

http://www.cairn.info/revue-rimhe-2013-3-page-94.htm

Pour citer cet article :

Chanut Odile et Paché Gilles, « La culture de mutualisation du PSL peut-elle favoriser l'émergence d'une logistique urbaine durable ? »,

RIMHE, 2013/3 n7, p. 94-110.

Distribution électronique Cairn.info pour ARIMHE.

(C) ARIMHE. Tous droits réservés pour tous pays.

La reproduction ou représentation de cet article, notamment par photocopie, n'est autorisée que dans les limites des conditions générales d'utilisation du site ou, le cas échéant, des conditions générales de la licence souscrite par votre établissement. Toute autre reproduction ou représentation, en tout ou partie, sous quelque forme et de quelque manière que ce soit, est interdite sauf accord préalable et écrit de l'éditeur, en dehors des cas prévus par la législation en vigueur en France. II est précisé que son stockage dans une base de données est également interdit. 


\title{
La culture de mutualisation du PSL peut-elle favoriser l'émergence d'une logistique urbaine durable?
}

\author{
Odile $\mathrm{CHANUT}^{1}$ et Gilles PACHÉ ${ }^{2}$
}

\begin{abstract}
Résumé
La métropolisation, tout comme l'urgence sociétale du développement durable, obligent à repenser les schémas de logistique urbaine de manière à limiter les effets néfastes de la livraison des marchandises en centre ville, tout en intégrant les évolutions récentes du commerce (retour au commerce urbain de proximité et croissance de l'Internet marchand). La mutualisation permet de relever un certain nombre de défis et se présente comme une manœuvre collective incontournable, au service d'objectifs économiques et sociétaux inscrits dans une logique de développement durable. La question posée dans cet article est celle du rôle possible des prestataires de services logistiques (PSL), en tant qu'acteur tiers, pour parvenir à faire travailler ensemble des acteurs habitués à gérer séparément leurs chaînes logistiques et imaginer de nouveaux schémas de logistique urbaine laissant une place importante à la mutualisation des ressources entre entreprises éventuellement concurrentes. Le PSL bénéficie, à ce niveau, d'une culture de mutualisation forte qu'il devrait être capable de décliner dans un grand nombre de contextes. La force des PSL est d'avoir très vite compris qu'en situant à l'interconnexion de plusieurs chaînes logistiques, ils s'avèrent capables de mutualiser des ressources en faisant ainsi jouer un double phénomène d'économies d'échelle et d'apprentissage. Ils pourraient jouer un rôle clé pour lever les freins possibles à la coopétition, mode relationnel qui semble favorable au contexte actuel du commerce de centre ville (position relative forte des chaînes de distribution et besoin fort en ressources externes), à la condition toutefois d'acquérir les compétences nécessaires à la gestion de capillarité pour le dernier kilomètre.
\end{abstract}

Mots clés : Culture, développement durable, logistique urbaine, mutualisation, prestataire de services logistiques (PSL).

\begin{abstract}
The fact that competitive manufacturing or retailing companies share resources to enhance their operations is now a well known reality. This is especially true for logistical resources necessary for the delivery of products to the customer; in this specific case, resources as transport and warehouses are increasingly pooled among companies. This article investigates the role of third party logistics service providers (3PLs) to achieve a shared management of logistical resources. At this level, the 3PLs take advantage of a strong culture of pooling that they should be able to use in many contexts, including urban logistics. The 3PLs quickly understood that their situation at the interconnection of several supply chains creates good conditions for pooling resources, using a dual phenomenon of economies of scale and economies of learning. Pooling is a collective strategy pursuing economic and societal goals in a sustainable development perspective. The objective of this article is to question the favorable strategic positioning of 3PL in the context of new urban retail formats. The capillarity imposed by convenience stores, inside the town, demands the rethinking of the organization of urban logistics by implementing pooling procedures to avoid traffic jams inside towns: should 3PL play a key role in this context?
\end{abstract}

Key words: Culture, sustainable development, urban logistics, pooling, third party logistics service provider (3PL).

1 - Professeur en Sciences de Gestion, Aix-Marseille Université, Centre de Recherche sur le Transport et la Logistique (CRET-LOG) - odile.chanut@univ-amu.fr

2 - Professeur en Sciences de Gestion, Aix-Marseille Université, Centre de Recherche sur le Transport et la Logistique (CRET-LOG) - gilles.pache@univ-amu.fr 
La métropolisation est désormais un phénomène de grande ampleur, à l'échelle de la Planète, ce qui signifie que la ville est le point de rencontre d'une multiplicité de flux entrants (par exemple, les produits de consommation) et de flux sortants (par exemple, les déchets). Wiskerke et Viljoen (2012) nous rappellent une réalité que d'aucuns oublient parfois: le nombre de personnes habitant dans des zones urbaines sera d'environ 6,5 milliards d'ici 2050, soit 200.000 bouches supplémentaires à nourrir chaque jour dans les quarante prochaines années! Sans un minimum de coordination logistique, l'espace urbain risque rapidement d'être encombré, voire saturé, en générant des nuisances d'une telle intensité qu'elles finiront par rendre insupportable la vie des résidants. Il n'est donc pas surprenant que les collectivités locales soient désormais attentives à une meilleure organisation du fret en milieu urbain dans un objectif de durabilité, tout en maintenant la vitalité économique de la ville (Anderson et al., 2005). En d'autres termes, la perspective « développement durable » doit s'articuler avec le respect de contraintes économiques et managériales en termes de performance des entreprises impliquées dans la gestion des flux entrants et sortants précités. Contrairement à ce que certains observateurs pensent, il ne s'agit pas d'une prise de conscience récente puisque, dès le milieu des années 1990, Hesse (1995) s'interrogeait sur l'imbrication problématique entre espace urbain et logistique.

L'objectif du présent article est d'aborder de front la question d'une logistique urbaine plus durable. En effet, la coordination des flux entrants et sortants précités exige de penser des schémas de nature collective en évitant que les égoïsmes individuels, autrement dit les politiques concurrentielles conduites par chaque entreprise pour accroître sa part de marché, conduisent à une sorte d'anarchie organisationnelle dont les premières victimes seraient les résidants de la ville. Si les défis managériaux demeurent à l'évidence prégnants, cette interpellation nous situe aussi et surtout dans la filiation du projet humaniste tel qu'annoncé par Brasseur et ses coauteurs (2012, p. 4): « placer l'Homme au centre de l'analyse des pratiques et modèles de management». La thématique que nous abordons est celle du rôle privilégié que pourraient tenir, dans de nouveaux schémas de logistique urbaine à inventer, les prestataires de services logistiques (dénommé par la suite PSL).

Le PSL est un puissant acteur des chaînes logistiques, apparu depuis une trentaine d'années et très présent en Europe, tout particulièrement en France. Nouvel entrant, il s'est doté de compétences "pointues" qui ont abouti à la mutualisation de ressources entre concurrents, que la compétition s'exerce entre eux au plan vertical (entre un industriel et un distributeur, par exemple) ou au plan horizontal (entre deux distributeurs, par exemple). Rien ne plus naturel aujourd'hui que de voir un PSL gérer, sur un mode mutualisé, les stocks de produits finis de trois distributeurs en concurrence frontale, et livrer ensuite leurs magasins avec des tournées dédiées ou partagées. Par-delà les enjeux économiques liés aux mutualisations en cours des ressources logistiques, se dessinent des enjeux écologiques majeurs en termes de réduction de l'empreinte carbone, auxquelles les villes sont de plus en plus sensibles pour améliorer le bien-être de leurs résidants. L'expérience acquise par les PSL, le « substrat culturel » développé en matière de stratégies collectives de mutualisation, ne pourraient-ils pas être dupliqués à l'échelle urbaine? La question mérite d'être posée.

Pour y répondre, nous nous appuierons sur les « traces » qu'il est possible d'identifier au sein de la prestation de services logistiques en France, principalement en référence à deux 
sources d'information: des contacts réguliers avec l'un des trois principaux PSL français dans le cadre de recherches conjointes ayant conduit à la publication de plusieurs travaux académiques; une collaboration avec les membres de la Confédération Française du Commerce Interentreprises, organisme finançant, au travers d'une chaire, des recherches portant sur l'intermédiation en contexte de logistique urbaine. La présente contribution revêt par conséquent une dimension réflexive sur la base de signaux faibles, au sens d'Ansoff (1975), en matière de stratégies logistiques durables, auxquelles s'associent de plus en plus systématiquement les villes. L'article est organisé en deux parties. Dans un premiers temps, selon une visée compréhensive, nous fournirons quelques éléments sommaires afin de mieux appréhender la prestation de services logistiques et la logique de mutualisation qui la structure. Ceci permettra, dans un second temps, de souligner en quoi la « culture » de certains PSL, les plus avancés en termes d'innovation organisationnelle, les rend sans doute aptes à répondre aux défis économiques et environnementaux de la logistique urbaine.

\section{Comprendre la prestation de services logistiques}

Il suffit de consulter régulièrement la presse professionnelle spécialisée en distribution et en logistique pour voir relatées de façon récurrente des success stories reposant sur un partenariat actif entre tel industriel (ou distributeur) et son PSL, à l'image de la relation symbiotique nouée de longue date entre l'enseigne McDonald's et MartinBrower (ex-LR Services) en France. Pour en comprendre les défis actuels et futurs, un effort de clarification préalable s'impose. Loin de l'image d'Épinal qui les confine à des transporteurs améliorés, les PSL ont su élargir leur système d'offre de manière significative, en mutualisant de manière performante des ressources, d'abord au niveau du transport (gestion des acheminements), puis au niveau de l'entreposage et du stockage (gestion des flux), enfin au niveau de la personnalisation de masse (gestion des réseaux de chaines logistiques). Nous reprendrons ici la définition du PIPAME (2009, p. 38) pour lequel la mutualisation des ressources logistiques consiste en « la mise en commun volontariste de moyens physiques, d'informations et de compétences dans le but d'obtenir à long terme des gains économiques, écologiques, financiers et/ou d'image, ou bien encore, de parer à court terme à une contrainte. Le cadre de cette coopération peut revêtir des formes juridiques et organisationnelles variables en fonction de la nature des parties, des moyens et des produits ou services $»$.

\subsection{Par-delà le transport: un système d'offre complexe}

Si l'on se place dans une perspective historique, les entreprises manufacturières ont été, de longue date, des adeptes d'une logistique de distribution en moyens propres. Ainsi, dès les années 1920, de nombreux fabricants manifestent la volonté d'organiser leur structure commerciale en abandonnant les grossistes et négociants indépendants, accusés d'entraver la mise en œuvre d'une politique de vente active. Étudiant le cas des eaux embouteillées en France, Marty (2008) souligne combien les grossistes entrepositaires resteront présents jusqu'aux années 1960, avant que l'emballage perdu (sans consignation) ne soit massivement adopté par les industriels. La rationalisation logistique ne sera pas à l'ordre du jour avant plusieurs décennies chez ces derniers, mais la possession d'entrepôts régionaux, bien qu'ayant une aire 
de marché assez étroite, permet alors de toucher les distributeurs sans se heurter à l'écran opaque que constituent les intermédiaires.

De leur côté, et pratiquement au même moment, quelques groupes succursalistes français comprennent tout l'intérêt de prendre le contrôle de la fonction de gros. L'augmentation significative des capacités de stockage de marchandises (et donc d'achat) donne en effet la possibilité d'accroître leur pouvoir de négociation vis-à-vis des fournisseurs en s'approvisionnant directement auprès d'eux. Là encore, l'option choisie est celle de la logistique patrimoniale, faute d'une offre d'entreposage locatif relativement performante, à l'exception de quelques Magasins Généraux de province. La tendance s'inverse dans le courant des années 1970, dans un premier temps au Royaume-Uni. En effet, de très nombreux distributeurs britanniques ayant pris le contrôle de la fonction de gros commencent à se tourner vers des PSL pour assurer l'ensemble des tâches liées à l'approvisionnement des points de vente. On peut identifier Exel Logistics comme étant le premier PSL moderne, à la suite du montage d'une opération de partenariat avec Marks \& Spencer au milieu des années 1970.

Dans sa dixième édition des termes de la logistique publié en octobre 2011, la revue professionnelle Logistiques Magazine donne une définition générale, mais suffisante en première approximation, du PSL: il s'agit d'une entreprise assurant la réalisation d'activités logistiques pour le compte d'un industriel ou d'un distributeur. Il n'y a pas de transfert de titres de propriété entre le PSL et son donneur d'ordre, ce qui le distingue ainsi du grossiste qui, quant à lui, sélectionne un assortiment de produits, puis les achète aux producteurs, pour les revendre à des utilisateurs professionnels (dont les distributeurs) et à des collectivités. Les deux acteurs exercent cependant des fonctions similaires en matière d'allotissement, de stockage et d'éclatement, expliquant peut-être la confusion dont ils sont souvent l'objet. Trois principales familles de PSL sont présentes sur le marché, en fonction de la complexité du système d'offre:

- les PSL classiques. Issus pour la plupart du secteur des transports routiers, ils assurent la simple exécution d'opérations physiques liées au transport, à la manutention et au stockage des produits intermédiaires ou finis de l'entreprise cliente;

- les PSL à valeur ajoutée. Ils intègrent en sus la gestion d'opérations à caractère industriel ou commercial (par exemple, différenciation retardée), à caractère administratif (par exemple, facturation) et à caractère informationnel (par exemple, tracking-tracing);

- les PSL dématérialisés. Ne disposant quasiment pas de moyens physiques propres, ils construisent leur prestation en mobilisant des ressources auprès de sous-traitants spécialisés, et en assurent la cohérence par une totale maîtrise des flux d'information.

Les deux premières familles sont fréquemment regroupées sous l'appellation de third party logistics (3PL) et la troisième sous celle de fourth party logistics (4PL). Cette dernière famille, la plus récente, est sans doute la moins développée, puisque les premières organisations de ce type apparaissent dans le courant des années 1990. Leur métier consiste à concevoir et à vendre des solutions logistiques sur mesure en créant une sorte de réseau de compétences qui associe transporteurs, entrepositaires, 
façonniers industriels, etc. Le système d'offre est composé, décomposé et recomposé à la demande en référence au modèle " plug and play/ unplug » introduit par FabbeCostes (2005). Au fil du temps, l'industrie de la prestation logistique au sens large, regroupant à la fois les $3 \mathrm{PL}$ et les $4 \mathrm{PL}$, a pris une importance grandissante dans les économies occidentales. Elle apparaît assez hétérogène pour au moins deux raisons: d'une part, y convergent des entreprises qui ont des appartenances sectorielles diverses (transporteurs routiers, ex-grossistes, filiales logistiques de distributeurs et d'industriels, etc.); d'autre part, les exigences des chargeurs sont de plus en plus singulières en fonction des caractéristiques des familles de produits commercialisées.

Globalement, une prestation de services logistiques peut comprendre tout un ensemble de composantes modulaires susceptibles d'être choisies par les expéditeurs et/ou les destinataires des marchandises, c'est-à-dire les industriels et/ou les distributeurs, et assemblées en fonction de leurs besoins singuliers (Fulconis et al., 2011). Dit autrement, la logique d'action du PSL est celle d'un assembleur dynamique de ressources et compétences, que l'on retrouve dans l'approche des «bundling logistics service providers » de Delfmann et ses coauteurs (2002). Les composantes modulaires, qui définissent les contours du métier, se regroupent traditionnellement en quatre catégories:

- les opérations de transport et auxiliaires: groupage / dégroupage, organisation des tournées de livraison, location de véhicules avec ou sans chauffeur, etc.;

- les opérations techniques de distribution physique: manutention, préparation de commandes, reconditionnement, étiquetage et marquage des prix, etc.;

- les opérations de gestion proprement dites : entreposage et tenue des stocks, gestion des dates de péremption, traitement des commandes, etc.;

- les opérations à caractère industriel ou commercial: facturation, gestion du service après-vente, assemblage terminal et bancs d'essai, etc.

Toutes ces opérations n'ont pas la même importance stratégique, ni le même potentiel de croissance sur le marché. Ainsi, les activités traditionnelles de transport (et auxiliaires) sont une composante que les PSL préfèrent souvent confier en soustraitance à de petits transporteurs routiers, placés dans une relation de dépendance, dans la mesure où l'acheminement n'est que faiblement différenciateur et peu rémunérateur. À l'opposé, d'autres services connexes, à haute valeur ajoutée, sont considérés comme des axes majeurs de développement, transformant certains PSL en des sortes d'ensembliers proches de façonniers industriels. L'encadré 1 donne un exemple de cette surprenante évolution en référence au système d'offre de Norbert Dentressangle, notamment en matière de co-packing. On retrouve ici, sur un registre singulier, le concept de plate-forme modulaire développé par Sanchez et Mahoney (1996), et qui constitue le fondement d'une économie de la modularité en cours de construction. 
Encadré 1: L'offre modulaire de Norbert Dentressangle

(http://www.norbert-dentressangle.com)

De l'assemblage à l'étiquetage, Norbert Dentressangle est en mesure d'intervenir sur les produits réceptionnés en entrepôts, en température ambiante ou dirigée. Objectif: apporter une valeur ajoutée au produit initial. L'entreprise assure la préparation des commandes et intervient sur les produits de façon plus ou moins complexe:

- assemblage simple;

- kitting (assemblage jusqu'à six composants différents, notamment pour le secteur de la téléphonie mobile);

- préparation des produits selon les «recettes» des industriels pour le secteur de l'agroalimentaire, emballage (mise sous film, reconditionnement à façon, mise sous box); - étiquetage (en braille, dans toutes les langues).

Parallèlement, l'entrepôt peut proposer des services d'échantillonnage et des tests qualité. Pour les actions promotionnelles de ses clients, Norbert Dentressangle est en mesure de fabriquer un nouveau produit à partir de plusieurs composants: c'est le co-packing. Pour ces opérations, l'entreprise automatise certaines opérations techniques ou fait appel à ses équipes pour les opérations simples, comme la mise en box. Exigeant des délais courts, ces opérations nécessitent par conséquent une gestion serrée des plannings. Pour l'entreprise, le co-packing réalisé par le PSL présente trois avantages:

- disposer d'un entrepôt unique pour le stockage et pour l'assemblage;

- réduire les délais et les coûts de façon significative en évitant les transports d'un site à l'autre;

- augmenter la réactivité et la flexibilité de la préparation de commande.

\subsection{La mutualisation, une " affaire " de PSL?}

L'idée dominante, largement partagée par les observateurs attentifs du fonctionnement des chaînes logistiques, repose sur le fait que les PSL ont su accompagner les stratégies de recentrage des industriels et des distributeurs sur leurs compétences fondamentales: la conception de nouveaux produits et services, la communication publicitaire autour de puissantes marques ou enseignes, le développement de techniques de vente originales, etc. L'intelligence stratégique de certains PSL fut d'anticiper puis de satisfaire les attentes beaucoup plus sophistiquées qui se sont manifestées depuis lors, dans l'industrie et le commerce, pour des services à haute valeur ajoutée. Cette intelligence stratégique, les rendant capables d'affirmer un avantage concurrentiel durable vis-à-vis de leurs clients, est en grande partie fondée sur la capacité d'identifier des « nœuds » de chaînes logistiques, c'est-à-dire des points fixes d'articulation entre différentes séquences de transport (entrepôt, plate-forme, etc.) au sens de Raimbault et ses coauteurs (2010), qui revêtent des caractéristiques suffisamment communes pour être pilotés de manière globale, et non chaîne par chaîne; on pourra prendre l'exemple d'un entrepôt de produits frais mis en place pour le compte de plusieurs distributeurs concurrents. De manière précoce, les PSL ont ainsi démontré tout l'intérêt de tournées de livraison communes à plusieurs réseaux de magasins pour réduire les coûts d'approvisionnement. Plus tard, ils prouveront, sur le même registre, l'intérêt d'une gestion mutualisée des stocks de matières et marchandises, là où certains modèles stratégiques prônaient une sauvegarde des sources de l'avantage concurrentiel par un cloisonnement des systèmes logistiques entre concurrents.

Pour affirmer leur avantage concurrentiel, les PSL, du moins les plus puissants des 3PL, ont rapidement construit des capacités d'entreposage de très grande taille (plusieurs centaines de milliers de $\mathrm{m}^{2}$ ). Elles sont effectivement garantes de phénomènes d'échelle 
ayant un pouvoir d'attraction très important sur de nouveaux clients potentiels, attirés par de faibles coûts unitaires de transport et/ou de gestion des stocks. La force des PSL est ainsi d'avoir très vite compris qu'en se situant à l'interconnexion de plusieurs chaînes logistiques, par exemple en gérant des entrepôts communs à plusieurs distributeurs, ils s'avèrent capables de mutualiser des ressources en faisant jouer un double processus d'économie d'échelle et d'apprentissage. La mutualisation impulsée par les PSL se présente ainsi comme une manœuvre collective incontournable, au service d'objectifs économiques, mais aussi sociétaux. Objectifs sociétaux dont la forte dimension durable n'échappe plus, et qui révèle de ce fait une évidente proximité avec les approches humanistes (Paradas, 2012). Les expériences de mutualisation peuvent être qualifiées de verticales lorsqu'elles aboutissent à la mise en commun de moyens entre entreprises situées à des niveaux différents du canal de distribution, par exemple entre un industriel $\mathrm{X}$ et un distributeur $\mathrm{Y}$, ou entre un franchiseur et ses franchisés (Chanut et al., 2011). Elles peuvent être qualifiées d'horizontales lorsqu'elles impliquent des entreprises ou groupes d'entreprises directement concurrentes au même stade de la chaîne logistique, par exemple entre trois enseignes de distribution ou entre deux industriels de l'agroalimentaire; on parle dans ce cas de démarche de pooling.

Incontestablement, les PSL tiennent une place de choix dans les mutualisations verticales et, plus récemment, horizontales. D'un point de vue historique, leur savoir-faire s'est déployé de manière privilégiée le long des chaînes logistiques, en associant plusieurs magasins autour d'infrastructures logistiques communes. Les PSL ont ainsi pu démontrer leurs aptitudes à impulser des logiques de massification des flux de plus en plus fortes sur quelques nœuds logistiques judicieusement localisés dans l'espace, au barycentre des magasins à desservir, pour bénéficier d'un prix du sol très attractif loin des villes. Depuis le milieu des années 2000, de nouveaux schémas logistiques d'approvisionnement se sont multipliés dans le secteur de la distribution. Ils reposent sur une démarche de pooling associant deux séquences connectées: dans un premier temps, les industriels livrent un entrepôt multi-fournisseurs (ou entrepôt primaire) au sein duquel les flux sont massifiés; dans un second temps, les industriels livrent - à plusieurs - les entrepôts de distributeurs (ou entrepôts secondaires) en fonction de leurs besoins, entrepôts qui livreront ensuite les magasins. Sont ainsi mutualisés, entre industriels parfois concurrents directs, le transport, le stockage, voire la gestion des approvisionnements en amont de l'entrepôt du distributeur (Hiesse et Paché, 2010).

Le management des chaînes logistiques en devient globalement plus efficace et plus efficient, tout particulièrement au niveau du transport. Il ne pouvait en aller autrement pour des raisons de compétitivité économique, compte tenu de l'évolution tendancielle de la structure des coûts logistiques au sein des entreprises européennes. L'European Logistics Association (ELA), en collaboration avec AT Kearney, fait en effet ressortir une augmentation significative depuis le milieu des années 2000, après une longue période de baisse, des coûts logistiques directement corrélée aux coûts de transport (voir la Figure 1). Mieux gérer les transports, notamment par un partage de mêmes véhicules entre concurrents, devient inévitable. Rien de véritablement original si l'on suit les travaux précurseurs de Bonet (2000), qui ont mis en avant l'importance de collaborations logistiques dans les canaux de distribution pour parvenir à plusieurs à l'obtention d'un avantage concurrentiel durable. 
Figure 1: Évolution des coûts logistiques en Europe de 1987 à 2012

(ELA/ AT Kearney)

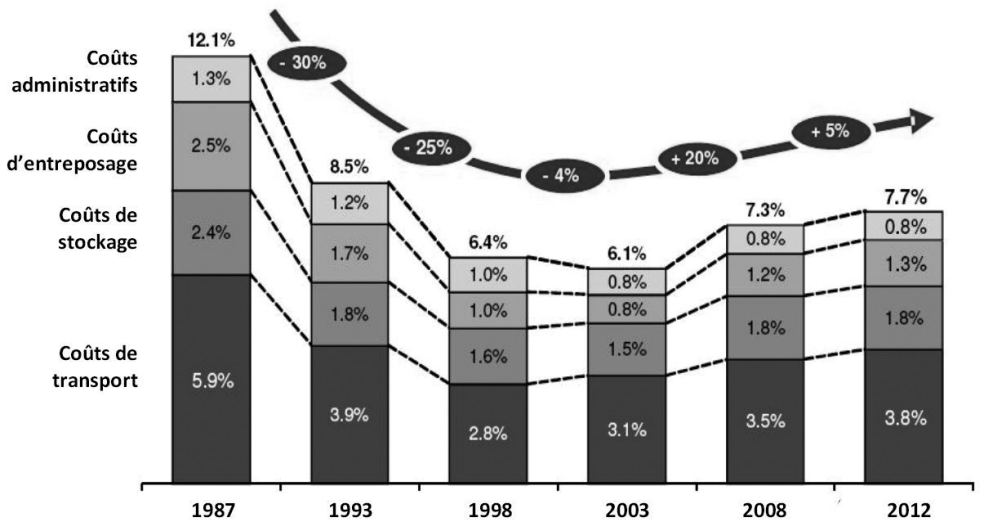

Le pooling en matière de transport, mais aussi d'entreposage, est bien illustré par le partenariat conclu entre Pepsi-Cola et d'Orangina, qui ont développé en France un cocamionnage de ramassage auprès des différents centres d'embouteillage, à destination des entrepôts du distributeur Auchan, leur client commun. Outre d'importantes économies d'échelle en matière de transport amont, liées à l'hyper-massification des flux, il en résulte un taux de remplissage largement accru qui permet de diminuer de manière significative l'empreinte carbone liée à l'usage du transport routier. D'autres expériences de pooling sont en cours, qui incluent parfois en outre des solutions de transport innovantes, multimodales, avec l'usage des voies ferroviaires ou fluviales, dont l'empreinte carbone au kilomètre parcouru reste beaucoup plus faible que la route. Couplées aux pratiques de mutualisation des entrepôts, de telles démarches ont pour vertu de réduire le risque tout en améliorant l'éco-efficience des systèmes logistiques (Franklin et Spinler, 2011). C'est surtout au niveau de la logistique urbaine que les mutations induites par le pooling apparaissent comme les plus profondes, et en partie irréversibles. Afin d'éviter l'asphyxie des espaces urbains, les collectivités territoriales sont désormais actives pour impulser de nouveaux schémas de logistique urbaine, laissant une part importante à la coopération et la mutualisation.

\section{Quelle place pour le PSL dans la logistique urbaine?}

Le commerce de détail connait depuis plusieurs années une rapide évolution. Elle est marquée principalement par un retour en force des magasins de proximité en centre ville, sur un modèle très connu au Japon (Capo et Chanut, 2012), le développement rapide du commerce électronique et l'essor continu des réseaux de franchise, également très présents, de longue date, en centre ville. Cette évolution se traduit par une rupture avec une tendance séculaire ayant conduit la distribution de masse à localiser ses magasins, de type super et hypermarchés, dans des zones péri-urbaines, et à jouer sur des phénomènes de massification extrême des flux d'approvisionnement, notamment par camions complets. Les nouveaux modèles émergents privilégient au contraire des 
logiques de « capillarité » compte tenu des points de destination ultime des produits. Ceci introduit des problématiques extrêmement complexes de circulation des marchandises dans la ville (Boudouin, 2006). Et cela, dans un contexte sociétal qui érige désormais comme priorité une ville durable mettant l'accent sur la qualité de l'air et la qualité de vie dans ses murs. L'une des mutations les plus significatives est sans doute la volonté des collectivités locales de planifier la mise en œuvre de structures collectives de transbordement des produits pour réduire l'engorgement des infrastructures urbaines. La mutualisation obligée des ressources qui en résulte offre-t-elle l'opportunité aux PSL d'y dupliquer leur savoir-faire accumulé par le passé?

\subsection{La logistique urbaine, un terrain privilégié de mutualisation horizontale}

De nombreux pays développent depuis une dizaine d'années des expériences de mise en place de systèmes de livraison urbaine dont l'objectif est la mutualisation des opérations d'entreposage de produits et l'optimisation des tournées à destination des magasins d'une zone donnée (Quak et de Koster, 2009; Guyon et al., 2010; Stumm et Kidd, 2012). La question centrale est désormais de les « industrialiser », c'està-dire de les faire passer du statut d'expérience à celui de modèle d'organisation à visée universaliste dans le cadre d'une "city logistics» (Whiteing et al., 2003; Petersen, 2006). Le but recherché est de diminuer le nombre de trajets de véhicules de livraison, de créer des possibilités élargies d'utilisation de matériels moins polluants, et de mieux gérer l'occupation d'un espace public de plus en plus encombré. Sur le plan fonctionnel, une telle organisation se traduit souvent par le regroupement sur une plate-forme unique, appelée Centre de Distribution Urbaine (CDU), des marchandises à destination de la zone à livrer. Le dernier kilomètre est alors assuré depuis le CDU, avec des véhicules légers, moins polluants et moins encombrants. L'exemple des magasins Sephora en France est une excellente illustration des mutations en cours (voir l'encadré 2).

\section{Encadré 2: Sephora et les enjeux de la ville durable}

(Supply Chain Magazine, mars 2010)

Inéluctablement, il se passera tôt ou tard en France ce que l'on voit poindre dans d'autres grandes villes à l'étranger: la limitation des accès au centre ville par des transports polluants. Sephora, un distributeur spécialisé dans la vente de parfums, cherchait des solutions techniques à cette problématique de long terme lorsque Deret, le PSL avec lequel il travaillait depuis des années, a présenté courant mai 2009 son projet de mettre en place 22 platesformes suburbaines en France, avec une livraison du dernier kilomètre en camion électrique. Pour cela, Sephora devait modifier profondément ses horaires de réapprovisionnement des magasins et son plan de transport national. La décision a été prise début juin 2009. Toutes les plates-formes suburbaines étaient opérationnelles six mois après. Le bilan est très positif, avec plus de souplesse et de réactivité en cas d'aléas, comme les chutes de neige que la France a connu juste avant Noël 2009. Au total, 1.000 tonnes de $\mathrm{CO}_{2}$ sont économisées par an, à la fois sur le trajet final et sur l'approvisionnement des plates-formes suburbaines par camions complets. Au final, pour les dirigeants de Sephora, tout ce qui se livre en centre ville en petits volumes, de deux à quatre palettes, est éligible à un pooling.

L'archétype du CDU se présente, sur un plan opérationnel, comme une solution efficiente pour les PSL se partageant le marché de la livraison en ville. Le plus souvent, en effet, ils ne disposent pas, indépendamment les uns des autres, des quantités de produits permettant d'organiser leurs flux sur la base d'unités de transport 
optimisées (réalisation d'une tournée complète chaque jour, par exemple). En outre, les PSL sont confrontés à des contraintes de plus en plus lourdes (congestion, partage de la voirie, exigences des clients, etc.), qui pèsent sur leur choix de réaliser ou non par leurs propres moyens le dernier kilomètre, les restrictions imposées sur les mouvements de véhicules occupant d'ailleurs une place significative à ce niveau (Macharis et Melo, 2011). Le CDU est une alternative crédible à de telles contraintes; elle permet aux PSL qui le souhaitent de disposer d'une zone optimale de déchargement des marchandises à destination de la zone couverte par ledit CDU. De leur côté, pour les collectivités territoriales, les investissements immobiliers en matière de CDU sont un moyen de rationaliser les flux sur leur territoire, d'optimiser le chargement / déchargement des véhicules et d'organiser la desserte des magasins dans des créneaux horaires de moindre affluence. Penser la logistique urbaine, c'est ainsi se soucier d'un aménagement de l'espace dont la configuration même doit faciliter l'écoulement des flux de produits, sans oublier les flux de retour.

Doit-on considérer qu'il est dramatique pour les magasins de centre ville, appartenant à des réseaux contractuels ou à des réseaux intégrés, de partager le même PSL et de perdre ainsi une source d'avantage concurrentiel fondée sur l'excellence logistique développée par leur tête de réseau pour leur enseigne? Cela n'est pas sûr, à condition de prendre ses distances avec la doxa qui considère que la maximisation de la qualité de service logistique doit être recherchée. Or, le service logistique est seulement un élément parmi d'autres du système d'offre d'un distributeur, et son importance doit être relativisée dans son modèle d'affaires. Si la performance logistique est perçue comme un pré-requis minimal, comme une sorte de barrière à l'entrée que doit contourner toute entreprise pour espérer occuper une bonne place sur le marché, elle ne peut expliquer seule le niveau de satisfaction du client et n'est donc pas une condition suffisante de réussite. De fait, la planification de la logistique urbaine imposée aux entreprises par les collectivités locales, via les PSL gérant des ressources logistiques mutualisées, pourrait constituer une opportunité d'évolution positive. Effectivement, si le management des activités logistiques n'apparaît pas essentiel pour espérer fidéliser durablement les clients d'un magasin, il faut éviter pour le distributeur d'y consacrer massivement des ressources et des compétences, finalement beaucoup plus utiles et rentables pour les autres éléments du système d'offre (par exemple, la communication publicitaire ou la formation des vendeurs).

La logistique urbaine renvoie finalement à des enjeux autant managériaux que sociétaux. Certes, il serait maladroit de sous-estimer les enjeux managériaux liés à une optimisation des déplacements de marchandises dans la ville. Comme on l'a souligné, l'amélioration de la disponibilité des produits livrés aux clients conditionne directement un positionnement concurrentiel pour un ensemble d'entreprises en concurrence partageant astucieusement des ressources. Mais les enjeux sociétaux sont au moins aussi importants pour préserver les conditions de vie des générations futures, en les empêchant d'être confrontées à un environnement hostile, pollué et saturé de véhicules. Sait-on que le transport est le troisième plus important contributeur à la diffusion de gaz à effet de serre $(14,3 \%)$, à peine dépassé par l'électricité et la production de chaleur $(24,9 \%)$ et la production industrielle (14,7\%) (Franklin et Spinler, 2011)? On doit hélas reconnaître le retard en la matière au niveau de la nécessaire prise de conscience. La recherche de Lindholm (2010) confirme que la connaissance et la sensibilisation aux dimensions « durables » dans le 
domaine du transport urbain de marchandises restent extrêmement faibles, malgré les discours de façade; l'intérêt manifesté demeure marginal et l'on doit regretter la piètre implication des collectivités locales, peu enclines encore à considérer que la question de la logistique urbaine doit constituer une priorité absolue. L'initiative privée pourrait dès lors se positionner comme une alternative crédible, mais en articulation avec les objectifs de politique publique, dans une vision intégrative de la logistique urbaine: « Il est indispensable de penser la totale coopération de tous les acteurs qui influent sur la distribution du fret dans l'espace urbain, et d'assurer la cohérence de leur stratégies, tactiques et opérations » (Abassi et Johnson, 2012, p. 106).

\subsection{Une histoire de "culture"}

Au final, quelle place envisager pour le PSL dans le contexte actuel d'évolution des formes de distribution au sein de plusieurs pays d'Europe, caractérisées entre autres par un retour au commerce de centre ville, et par la croissance exponentielle de l'Internet marchand et des livraisons de produits alimentaires à domicile (Durand et GonzalezFeliu, 2012)? Force est de constater que l'expertise et la légitimité des PSL se sont appuyées sur des massifications de flux en partie contradictoires avec la capillarité qu'imposent les magasins de proximité et le commerce électronique. Parallèlement, on doit admettre que la capillarité oblige à repenser les schémas de logistique urbaine en imposant la mise en œuvre de procédures mutualisées pour éviter l'encombrement total des centres villes: les PSL ont certainement un rôle clé à jouer, à l'image du groupe Geodis à Paris (voir l'encadré 3).

Encadré 3 : Geodis, un PSL très impliqué dans la logistique urbaine (http://www.innovcity.fr)

$20 \%$ du trafic routier parisien est aujourd'hui le fait des camions de transport de marchandises, les poids lourds représentant $35 \%$ des émissions de $\mathrm{CO}_{2}$ en ville. De son côté, Geodis livre chaque jour dans la capitale 200 tonnes de marchandises à bord de 164 véhicules qui parcourent $8.560 \mathrm{~km}$. Un véritable casse-tête pour celui qui ose sortir la calculette à empreinte carbone. Le quatrième PSL européen s'y est attelé. C'est à Paris, dans le cadre de Distripolis, que l'offre a été lancée le 27 juin 2011. Le principe? Les marchandises, acheminées pour la plupart de nuit, sont livrées sur la plate-forme multimodale de fret de Bercy qui réceptionne à la fois trains, camions et bateaux. Une vingtaine de véhicules Electron prennent le relais trois à quatre fois par jour pour acheminer les marchandises vers les bases BLUE (acronyme de Base Logistique Urbaine Écologique). Bleue, c'est aussi la couleur de la nouvelle flotte de véhicules qui, à partir des bases BLUE, sert à acheminer les colis et palettes de plus de $200 \mathrm{~kg}$ jusqu'aux clients finaux. De petits triporteurs à assistance électrique ou des transpalettes et véhicules électriques légers livrent, quant à eux, les palettes et colis de moins de $200 \mathrm{~kg}$. Les premières bases BLUE ont fait leur apparition en 2012 à Bercy et Montparnasse. Huit bases du même type émergeront progressivement jusqu'en 2015. La nouvelle offre vise clairement à améliorer le dispositif de livraison du dernier kilomètre. Le plus complexe à gérer et le plus polluant, augmentée ces dernières années par le boom du commerce électronique. Une problématique à ce point universelle que le Groupement des Autorités Responsables de Transport (GART) a récemment lancé son club du dernier kilomètre de livraison. Les villes s'efforçant désormais de pousser les véhicules polluants hors de leurs enceintes, les PSL s'adaptent et innovent, notamment en déroulant le tapis rouge aux véhicules hybrides et électriques, plus maniables et rapides aux cœurs des villes saturées. Distripolis surfe donc sur cette vague pour diminuer son empreinte carbone. Et les objectifs sont clairs: une baisse de 1.747 tonnes de $\mathrm{CO}_{2}$ et particules émises, soit une diminution de $85 \%$ par rapport à aujourd'hui en 2015, lorsque toutes les bases parisiennes seront opérationnelles. Après Paris, Lille, Bordeaux et Strasbourg seront les prochaines villes françaises à accueillir des bases BLUE. 
En examinant les sources de l'avantage concurrentiel déployées par les PSL depuis leur émergence, jusqu'à leur expansion grâce à l'élargissement de leur champ d'expertise, il est possible d'identifier plusieurs éléments significatifs qui militent pour leur capacité à s'inscrire rapidement comme un acteur majeur dans les solutions de mutualisation à inventer pour la logistique urbaine. Ce faisant, les PSL devraient participer activement, et de manière efficace, à un usage de plus en plus parcimonieux des ressources logistiques, l'un des enjeux majeurs des prochaines années; l'enquête de Lieb et Lieb (2010) tend d'ailleurs à souligner l'implication croissante des PSL dans la poursuite d'objectifs « durables ». Les éléments significatifs précités renvoient à des dimensions que nous qualifierons de culturelles, en référence à la culture vue comme la manière de penser une action formalisée, acquise et partagée par une pluralité d'individus et/ou d'organisations, et qui constitue in fine les fondements d'une collectivité aux comportements globalement cohérents (Rocher, 1970). Dans le contexte de la prestation de services logistiques, les traits saillants à retenir, issus des recherches co-conduites avec l'un des principaux 3PL français, sont les suivants:

1) Les PSL ont une culture historique de la mutualisation, il s'agit du «noyau dur » de leur modèle d'affaires afin de rendre attractive leur offre en termes de coût unitaire. Mutualiser est une stratégie qui se positionne à l'origine des économies d'échelle et de champ dont ils sont les pourvoyeurs pour le compte de leurs clients. Le système d'approvisionnement centralisé des hyper et supermarchés, instauré dès le début des années 1980, se fonde sur des nœuds logistiques partagés par un nombre plus ou moins important d'enseignes. Les PSL ont donc appris très tôt à faire travailler ensemble des entreprises concurrentes en soulignant l'importance des massifications pour réduire les coûts unitaires d'approvisionnement des magasins, élément clé d'une stratégie de domination par les coûts.

2) Les PSL ont une culture technique de la mutualisation, ils savent développer des outils et des techniques de management facilitant l'agrégation des flux grâce à leur capacité incontestable à «modulariser» les opérations logistiques et à standardiser la gestion des interfaces pour une multiplicité de chaînes logistiques. Selon un phénomène d'apprentissage technologique bien connu, ils ont construit des savoir-faire qu'il est possible de dupliquer d'une chaîne logistique à l'autre puisqu'un investissement spécifique consenti pour un chargeur, par exemple en matière de préparation des commandes, conduit à assimiler un savoir-faire pouvant être adapté aux besoins actuels ou futurs d'un autre chargeur.

3) Les PSL ont une culture communicationnelle de la mutualisation, ils savent mémoriser leurs différences expériences, autant en matière de savoirs tacites que de savoirs formalisés, pour appliquer des solutions " clés en main » qui puisent dans un large registre de réponses antérieurement apportées à divers clients; comme le notent Cézanne et Saglietto (2011), on peut parler de capitalisation des connaissances acquises lors de l'exécution des différents contrats. Construire à la demande une chaîne logistique, y compris éphémère, s'appuie sur un agencement de modules (et/ou de compétences) dont la polyvalence est une caractéristique majeure. Les PSL les plus à l'avant-garde disposent ainsi, dans leur mémoire organisationnelle, d'un large registre de couplages entre modules, dont il suffira de modifier l'interfaçage en fonction des nouvelles demandes leur étant signifiées. 
4) Les PSL ont une culture juridique de la mutualisation, ils proposent à leurs clients d'externaliser sous de nouvelles conditions les opérations logistiques en se référant à des conventions collectives souvent plus attractives, et dissociées des conventions collectives rattachées au cœur de métier desdits clients. En outre, en maîtrisant parfaitement les différentes logiques contractuelles à l'œuvre, les PSL permettent à leurs clients chargeurs d'échapper au risque de condamnation pour pratiques anti-concurrentielles que soustend une approche coopétitive de la gestion des chaînes logistiques, entre autres dans le cadre des contrats partagés. L'acteur tiers qu'est le PSL, désormais apte à renégocier efficacement, à échéance, les contrats d'externalisation logistique (Avignon, 2009), fait ici travailler ensemble, et de façon collaborative, des concurrents frontaux qui conservent in fine leur indépendance juridique.

5) Les PSL, enfin, ont une culture sociétale de la mutualisation, ils s'insèrent dans de multiples réseaux sociaux (relationnels) compte tenu de leur activité qui les place à l'interconnexion d'un nombre important de secteurs, de familles de produits, de filières de production, etc., et qui sont autant de sources d'arbitrages entre entreprises concurrentes. Les contacts réguliers se nouent d'ailleurs au niveau de preneurs de décision qui apprennent à se connaître, qui développent entre eux des affects positifs, voire des empathies, propices à la mise en œuvre de partenariats durables; la dimension humaine relative au succès de chaînes logistiques collaboratives constitue une thématique de recherche aujourd'hui importante (Borgatti et Li, 2009; Galaskiewicz, 2011).

En d'autres termes, tout porte à croire que les PSL innovants sont capables de lever sans difficulté les freins possibles à la coopétition, comme ils ont appris à le faire de manière émergente, et de plus en plus délibérée au fil du temps, en agissant pour le compte d'une pluralité de chargeurs. Pour comprendre cette position, il est nécessaire de se référer à la contribution de Bengtsson et Kock (1999), qui précise les conditions nécessaires à la mise en œuvre d'un mode relationnel de type coopétition: les concurrents doivent occuper une position relative forte sur le secteur d'activité (ce qui exclut toute alliance de nature "asymétrique»), et le besoin en ressources extérieures doit être élevé (ce qui exclut une situation structurellement déséquilibrée de dépendance vis-à-vis des ressources). Lorsque ces deux conditions ne sont pas remplies, les modes relationnels à privilégier sont la coopération, la compétition ou la coexistence (voir le Tableau 1). Or, dans le contexte actuel de la logistique urbaine, les conditions de la coopétition sont réunies: on observe l'effondrement du commerce indépendant isolé et la montée en puissance des chaînes de distribution intégrées ou fédérées par de puissants franchiseurs (Boulay et Chanut, 2010). Ces chaînes sont désormais en position forte sur leurs secteurs; par ailleurs, leur besoin en ressources externes sont également forts, car elles doivent disposer d'une logistique parfaitement structurée obéissant à des exigences d'accès aux zones urbaines jamais connues par le passé.

Tableau 1: Modes relationnels entre entreprises concurrentes

(d'après Bengtsson et Kock, 1999, p. 180-182)

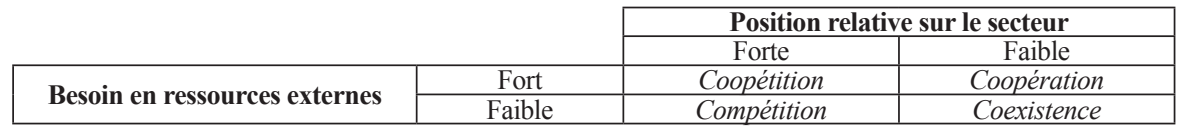


Le mode relationnel de la coopétition se présente ainsi comme le plus adapté pour le management des ressources dans l'espace urbain, en exigeant des entreprises concurrentes qui y pénètrent une mise en commun planifiée de moyens. Rien d'étonnant, si l'on suit le raisonnement de Bengtsson et Kock (1999), de remarquer que lorsque le commerce indépendant isolé était dominant dans les centres villes, avec des clientèles captives peu sensibles à l'excellence logistique, le mode relationnel de la coexistence était alors dominant, chaque magasin se tournant librement vers tel ou tel intermédiaire pour ses approvisionnements. Ce schéma est désormais caduc, et les PSL ayant développé une culture de la mutualisation peuvent se positionner comme des acteurs incontournables de la logistique urbaine. Il reste à savoir si ces derniers seront aptes, sur le plan contractuel, à exploiter cet avantage concurrentiel et, par dessus tout, s'ils disposeront d'un savoirfaire suffisamment adaptatif pour gérer la capillarité induite par les nouvelles logiques commerciales privilégiant la commodité et les livraisons dans des espaces urbains confinés. Au risque de voir de nouveaux entrants très dynamiques, ou des opérateurs historiques comme les grossistes, occuper rapidement le terrain...

L'autre interrogation majeure est relative à l'articulation entre les options retenues par les collectivités locales en matière de «gouvernance territoriale » et les stratégies de création de valeur poursuivies par les PSL. En effet, toute la difficulté résulte du fait que cette gouvernance, comme le notent Masson et Petiot (2010, p. 17), « doit être mixte, c'est-à-dire se réaliser dans le cadre d'un partenariat public-privé (....). L'infrastructure logistique relève à la fois du jeu du public, dans sa composante infrastructurelle mais aussi du jeu du privé dans sa composante servicielle». En d'autres termes, si les collectivités territoriales sont les porte-parole d'un bien commun, à savoir le bien-être collectif des résidants de la ville, les PSL ont aussi des comptes à rendre à un certain nombre de parties prenantes, au sein desquelles les actionnaires sont loin de jouer un rôle négligeable. Pour les collectivités locales, il s'agira donc de savoir arbitrer au mieux entre la nécessaire performance logistique, notamment pour continuer à rendre leur territoire attractif, et les exigences environnementales de développement durable (voir le Tableau 2). Il n'est pas sûr qu'un tel arbitrage puisse s'exercer aisément, tant les objectifs peuvent s'avérer contradictoires, par exemple entre massification des flux, à l'origine d'économies d'échelle, et nécessaire proximité pour une véritable logistique capillaire au service de l'Homme.

Tableau 2: Gouvernance territoriale, performance logistique et développement durable (d'après Masson et Petiot, 2010, p. 17)

\begin{tabular}{|l|l|}
\hline \multicolumn{1}{|c|}{ Performance logistique } & \multicolumn{1}{c|}{ Développement durable } \\
\hline Organisation spatiale coordonnée & Réduction des émissions polluantes \\
Articulation efficace des modes de transport & Création de sites multimodaux \\
Extension de la mutualisation & Usage de modes de transport alternatifs \\
Présence d'externalités d'agglomération & Couplage massification-capillarité \\
\hline
\end{tabular}

\section{Conclusion}

Il est désormais assez courant de reconnaître le rôle central tenu par les PSL dans la gestion des chaînes logistiques. Jusqu'à une période assez récente, les chargeurs industriels et commerciaux préféraient intégrer verticalement les activités logistiques pour « fiabiliser » le fonctionnement des filières de production. Tous les analystes ont encore en mémoire 
le cas d'école que constitue Tate \& Lyle, la plus puissante multinationale sucrière du monde, qui avait choisi jusqu' aux années 1970 de totalement contrôler les flux de matières premières, composants et produits finis, des plantations de canne à sucre dans les Antilles aux magasins d'alimentation au Royaume-Uni (Chalmin, 1983). On pourrait également citer le cas Michelin, qui a intégré l'ensemble de sa chaîne de valeur, des plantations d'hévéas pour le caoutchouc à la distribution avec sa filiale Euromaster, en passant par la conception et la fabrication des pneumatiques. Cet idéal-type de management appartient en grande partie au passé, même s'il demeure ponctuellement encore vivace ici et là. En effet, les chargeurs préfèrent désormais confier leur gestion logistique à des PSL, dont le champ d'intervention est de plus en plus étendu, en se recentrant sur leur cœur de métier. Les relations d'échange entre chargeur et PSL sont ainsi devenues un sujet de recherche particulièrement vivace depuis plus d'une vingtaine d'années; il mobilise pour cela des modèles d'analyse très variés issus du management logistique, mais aussi du marketing inter-organisationnel et du management stratégique.

En choisissant comme angle d'attaque privilégié la compétence centrale du PSL, à savoir sa capacité culturelle à mutualiser des ressources, nous avons souhaité indiquer que les politiques actuelles de mutualisation horizontale, tout particulièrement au sein des collectivités territoriales, pourraient y trouver un puissant appui opérationnel pour une meilleure gestion des marchandises dans la ville. Le débat est majeur pour certains pays, notamment latins, ayant pris du retard par rapport aux pays du Nord en matière de planification des flux de produits dans l'espace urbain. En effet, les expériences de logistique urbaine planifiée sont parmi les plus dynamiques en Allemagne ou aux PaysBas (Marinov et al., 2008), alors que Muñuzuri et ses coauteurs (2012) dressent un portrait catastrophiste des expérimentations conduites en Espagne, qui brille plus par le football que par sa discipline urbaine. À titre d'illustration, nous pourrions citer « le stationnement en double file dans le centre ville de Séville » (Muñuzuri et al., 2012, p. 140) qui reste sans conteste le sport préféré des chauffeurs des véhicules de livraison conduisant à une inévitable asphyxie urbaine. Les contraintes environnementales ne pourront que s'accentuer dans les prochaines années et il paraît évident que la liberté individuelle laissée à chaque entreprise d'implanter son propre système logistique n'y résistera pas. L'avenir ne serait-il pas, finalement, à la « ville frugale » (Haëntjens, 2012), qui réussit à produire plus de satisfaction avec moins de ressources? Si le diagnostic est imparable, encore faut-il se poser la question des acteurs les plus performants pour gérer les indispensables mutualisations sous-tendant la frugalité de demain, et la manière la plus efficace de les soutenir.

Les PSL les plus innovants, tout particulièrement les 3PL ayant assimilé une expérience « opératoire » de la mutualisation depuis près de trente ans, en s'occupant simultanément de la logistique d'une pluralité de grands distributeurs concurrents, possèdent sans aucune doute une compétence incontestable applicable au contexte urbain, à la fois en termes d'opérations de transport et auxiliaires et en termes d'opérations techniques de distribution physique. C'est la conclusion à laquelle nous pouvons raisonnablement arriver. Mais comme l'ont analysé Crozier et Friedberg (1977), dans une logique d'action stratégique, il ne faudrait pas oublier que le jeu des acteurs se développe en référence à un "système politique » structuré par des relations de pouvoir et d'influence. Si les PSL ont su s'enraciner dans des chaînes logistiques en jouant sur des phénomènes de massification péri-urbaine, et en ayant comme interlocuteurs des preneurs de décision 
qui s'appuient sur des modèles standard de choix des partenaires, tel n'est plus le cas de la logistique urbaine. En effet, les PSL sont face à des managers de ville dont la rationalité mixe objectifs économiques, écologiques et sociétaux. En d'autres termes, il s'agira ici de développer un dialogue constructif avec des collectivités locales d'abord soucieuses d'un aménagement harmonieux de la ville et du bien-être des résidants (au demeurant électeurs à échéance régulière...). Le PSL impliqué dans le fonctionnement d'une logistique urbaine mutualisée doit ainsi faire preuve de ses aptitudes à la formulation de solutions de synthèse en tant qu'agent d'interface, ou encore de « boundary spanner », dans l'espace de discussion noué entre public et privé. Cette perspective de recherche nous paraît urgente à investiguer et réclame que management public et management d'entreprise croisent leurs regards de manière plus incisive.

\section{Références}

Abassi M. et Johnson M. (2012), Themes and challenges in making urban freight distribution sustainable, in Carlsson C.-M., Emtairah T., Gammelgaard B., Vestergaard Jensen A. et Thidellp Å.(éds.), Rethinking transport in the Øresund region: policies, strategies and behaviours, Lund University \& Øresund EcoMobility, Lund, p. 93-111.

Anderson S., Allen J. et Browne M. (2005), Urban logistics-How can it meet policy makers' sustainability objectives? Journal of Transport Geography, Vol. 13, n 1, p. 71-81.

Ansoff H. (1975), Managing strategic surprise by response to weak signals, California Management Review, Vol. 18, n 2, p. 21-33.

Avignon S. (2009), La question de la durée et de l'adaptabilité des contrats d'externalisation: le point de vue d'un juriste, Logistique \& Management, Vol. 17, n 1, p. 19-30.

Barratt M. (2004), Understanding the meaning of collaboration in the supply chain, Supply Chain Management: An International Journal, Vol. 9, $\mathrm{n}^{\circ}$ 1, p. 30-42.

Bengtsson M. et Kock S. (1999), Cooperation and competition in relationships between competitors in business networks, Journal of Business \& Industrial Marketing, Vol. 14, n 3, p. 178-190.

Bonet D. (2000), Conflit et coopération dans le canal de distribution: une analyse du discours des acteurs, in Volle P. (éd.), Études et recherches sur la distribution, Economica, Paris, p. 231-246.

Borgatti S. et Li X. (2009), On social network analysis in a supply chain context, Journal of Supply Chain Management, Vol. 45, $\mathrm{n}^{\circ}$ 2, p. 5-22.

Boudouin D. (2006), Les espaces logistiques urbains, La Documentation Française, Paris.

Boulay J. et Chanut O. (2010), Les réseaux de franchise, La Découverte, Paris.

Brasseur M., Kefi H. et Ngijol J. (2012), Éthique et management: repenser l'organisation, Revue Interdisciplinaire sur le Management et l'Humanisme, $\mathrm{n}^{\circ}$ 1, p. 3-6.

Capo C. et Chanut O. (2012), Quand la proximité crée la convenience: une grille de lecture du système de distribution japonais, Les Cahiers Scientifiques du Transport, $\mathrm{n}^{\circ}$ 61, p. 91-117.

Cézanne C. et Saglietto L. (2011), Capital humain, prestataires de services logistiques et frontières de la firme, Revue d'Économie Industrielle, $\mathrm{n}^{\circ} 135$, p. 71-88.

Chalmin P. (1983), Tate \& Lyle, géant du sucre, Economica, Paris.

Chanut O., Capo C. et Bonet D. (2011), De la mutualisation verticale à la mutualisation horizontale: les enjeux et les critères de choix logistiques des réseaux sélectifs contractuels, Revue Française de Gestion Industrielle, Vol. 30, $\mathrm{n}^{\circ}$ 3, p. 37-59.

Crozier M. et Friedberg E. (1977), L'acteur et le système: les contraintes de l'action collective, Éditions du Seuil, Paris.

Delfmann W., Albers S. et Gehring M. (2002), The impact of electronic commerce on logistics service providers, International Journal of Physical Distribution \& Logistics Management, Vol. 32, n 3, p. $203-$ 222.

Durand B. et Gonzalez-Feliu J. (2012), Urban logistics and e-grocery: have proximity delivery services a positive impact on shopping trips? Procedia - Social and Behavioral Sciences, Vol. 39, p. 510-520.

Fabbe-Costes N. (2005), La gestion dynamique des supply chains des entreprises virtuelles, Revue Française de Gestion, n 156, p. 151-166. 
Franklin R. et Spinler S. (2011), Shared warehouses-Sharing risks and increasing eco-efficiency, International Commerce Review, Vol. 10, $\mathrm{n}^{\circ}$ 1, p. 23-31.

Fulconis F., Paché G. et Roveillo G. (2011), La prestation logistique: origines, enjeux et perspectives, Éditions Management \& Société, Caen.

Galaskiewicz J. (2011), Studying supply chains from a social network perspective, Journal of Supply Chain Management, Vol. 47, $\mathrm{n}^{\circ}$ 1, p. 4-8.

Guyon O., Absi N., Boudouin D. et Feillet, D. (2010), Plates-formes en centre ville pour la logistique urbaine: étude sur la ville de Marseille, Actes de la $2^{e}$ Journée de Recherche Mobilité, Transport et Logistique, Lyon, p. 1-8 (CD-rom).

Haëntjens J. (2012), Quel avenir pour la frugalité? L'exemple des villes, Futuribles, n 384, p. 35-43.

Hesse, M. (1995), Urban space and logistics: on the road to sustainability? World Transport Policy and Practice, Vol. 1, n 4, p. 39-45.

Hiesse V. et Paché G. (2010), Logistique mutualisée: une nouvelle architecture organisationnelle dans les canaux de distribution, Économies et Sociétés, Série Systèmes agroalimentaires, nº 32, p. 1513-1533.

Lieb K. et Lieb R. (2010), Environmental sustainability in the third-party logistics (3PL) industry, International Journal of Physical Distribution \& Logistics Management, Vol. 40, n 7, p. 524-533.

Lindholm M. (2011), A sustainable perspective on urban freight transport: factors affecting local authorities in the planning procedures, Procedia - Social and Behavioral Sciences, Vol. 2, n 3, p. 62056216.

Macharis C. et Melo S., éds. (2011), City distribution and urban freight transport: multiple perspectives, Edward Elgar Publishing, Cheltenham.

Marinov M., Zunder T. et Islam D. (2008), Urban freight consolidation concepts : is there something missing? Transport Problems, Vol. 3, $\mathrm{n}^{\circ}$ 2, p. 87-94.

Marty N. (2008), L'eau embouteillée: histoire de la construction d'un marché, Entreprises \& Histoire, $\mathrm{n}^{\circ} 50$, p. $86-99$.

Masson S., et Petiot R. (2010), Attractivité territoriale, infrastructures logistiques et développement durable, Actes du Colloque Joint ASRDLF-AISRe, Aoste, p. 1-20 (CD-rom).

Muñuzuri J., Cortés P., Guadix J. et Onieva L. (2012), City logistics in Spain: why it might never work, Cities, Vol. 29, n 2, p. 133-141.

Paradas A. (2012), L'homme comme sujet, Revue Interdisciplinaire sur le Management et l'Humanisme, n'2, p. 3-16.

Petersen, T. (2006), Development of a city logistics concept, Production Planning \& Control, Vol. 17, $\mathrm{n}^{\circ}$ 6, p. 616-623.

PIPAME (2009), Logistique mutualisée: la filière «fruits \& légumes » du Marché d'Intérêt National de Rungis, Ministère de l'Économie, de l'Industrie et de l'Emploi, Paris.

Quak H., et de Koster M. (2009), Delivering goods in urban areas: how to deal with urban policy restrictions and the environment, Transportation Science, Vol. 43, n 2, p. 211-227.

Raimbault N., Douet M., et Frémont A. (2010), Les plates-formes logistiques: entre fluidité et fixité, 4 Pages, Programme de Recherche FLUIDE, Agence Nationale de la Recherche, INRETS, Marne-laVallée.

Rocher G. (1970), Introduction à la sociologie générale. 1. L'action sociale, Éditions du Seuil, Paris.

Sanchez R., et Mahoney J. (1996), Modularity, flexibility, and knowledge management in product and organization design, Strategic Management Journal, Vol. 17, Special Issue, p. 63-76.

Stumm M., et Kidd J. (2012), Coordinating Parisian urban transport, in Chan, H.-K., Lettice, F., et Durowoju, O. (éds.), Decision-making for supply chain integration, Springer, Londres, p. 227-251.

Whiteing T., Browne M., et Allen J. (2003), City logistics : the continuing search for sustainable solutions, in Waters D. (éd.), Global logistics and distribution planning: strategies for management, Kogan Page, Londres, $4^{e}$ éd., p. 308-320.

Wiskerke J., et Viljoen A. (2012), Sustainable urban food provisioning: challenges for scientists, policymakers, planners and designers, in Viljoen, A., et Wiskerke, J. (éds.), Sustainable food planning: evolving theory and practice, Wageningen Academic Publishers, Wageningen, p. 19-35. 